\title{
Platinum-free cobalt ferrite based micromotors for antibiotic removal
}

Jemish Parmar, ${ }^{a, b}$ Katherine Villa, ${ }^{a}$ Diana Vilela, ${ }^{a, b}+$ and Samuel Sánchez ${ }^{a, b, c}+$

a. Institute for Bioengineering of Catalonia (IBEC), The Barcelona Institute of Science and Technology, Baldiri Reixac 10-12, 08028 Barcelona, Spain.

b. Max-Planck Institute for Intelligent Systems, Heisenbergstr. 3, 70569 Stuttgart, Germany

c. Institució Catalana de Recerca i Estudis Avançats (ICREA), Psg. Lluís Companys, 23, 08010 Barcelona, Spain

+Corresponding authors: dvilela@ibecbarcelona.eu (Vilela Diana); ssanchez@ibecbarcelona.eu (Samuel Sanchez)

Self-propelled micromotors have previously shown to enhance pollutant removal compared to non-motile nano-micro particles. However, these systems are expensive, difficult to scale-up and require surfactant for efficient work. Efficient and inexpensive micromotors are desirable for their practical applications in water treatment technologies. We describe cobalt-ferrite based micromotors (CFO micromotors) fabricated by a facile and scalable synthesis, that produce hydroxyl radicals via Fenton-like reaction and take advantage of oxygen gas generated during this reaction for self-propulsion. Once the reaction is complete, the CFO micromotors can be easily separated and collected due to their magnetic nature. The CFO micromotors are demonstrated for highly efficient advanced oxidative removal of tetracycline antibiotic from the water. Furthermore, the effects of different concentrations of micromotors and hydrogen peroxide on the antibiotic degradation were studied, as well as the generation of the highly reactive hydroxyl radicals responsible for the oxidation reaction.

\section{Introduction}

Self-propelled micro/nano motors have gained considerable attention because of their attractive practical applications and importance for understanding motion at low Reynolds number[1-5]. In recent years, researchers have demonstrated their use in sensing[6-8], micro surgery[9-11], drug delivery[12,13], and environmental applications[14,15]. Among these, the latter is particularly promising for near future practical applications of micromotors in waste water treatment[16-18]. Most of the micromotors-based systems reported for environmental applications are bubble propelled and contain platinum as a catalyst to obtain motion by decomposition of hydrogen peroxide into oxygen and water in the media. They are typically fabricated by various multi-step processes like template assisted synthesis or photolithography, and subsequent glancing angle deposition. These techniques produce multi-material structures with a cavity where bubbles can grow from the locally saturated oxygen and get ejected, which induces selfpropulsion. The motion of the catalytic self-propelled micromotors enhances micro-mixing and mass transfer in the aqueous system, thus increasing the removal rate of pollutants present in wastewaters by the functional material (adsorbent or oxidative catalyst) [15,19]. For instance, micromotors have been proven to be useful for heavy metal removal[20,21], organic removal[22-28] and bacteria disinfection[29-32]. However, several challenges must be addressed before micromotors-based water treatment technology can be used in large scale practical applications. One of the major challenges is the prohibitive cost associated with the use of platinum for micromotors propulsion[33]. Furthermore, current fabrication methods makes scaling up challenging and expensive[3]. The mass production method 
of micromotors based on an inexpensive material is the key to their large-scale viability for water treatment remediation. Efforts have been made to develop platinum free catalytic micromotors for organic removal from water, nevertheless the reported systems still require the addition of surfactants[34,35] to work efficiently. These surfactants are often toxic and add an extra organic contamination load in water.

Iron and other transition metals based react with hydrogen peroxide $\left(\mathrm{H}_{2} \mathrm{O}_{2}\right)$ via Fenton-Like reactions to produce reactive oxygen species (ROS), especially hydroxyl radicals (HO.) by decomposition of hydrogen peroxide. Fenton-Like processes are complex and include multiple chain reactions, which generate hydroxyl radicals and oxygen gas during the catalysis cycle[36]. Hydroxyl radicals generated by Fentonlike reactions are extremely oxidative, thus they are very important for advanced oxidative process to degrade refractory organic pollutants which cannot be removed naturally by microorganisms[37]. Pharmaceutical pollutants, namely antibiotics, hormones, and phenolic compounds are among the refractory pollutants that are toxic for humans and aquatic life even at very low concentrations[38]. Currently, industrial and municipal water treatment plants widely use separation and activated sludge methods to remove organic pollutants from wastewater, which is often not sufficient to remove these contaminants[39,40]. Various iron-based materials have been extensively studied for Fenton-like degradation of organic pollutants but their use for designing multifunction micromotors that can selfpropel and produce hydroxyl radicals is not yet well studied.

Here, we present platinum and surfactant free cobalt ferrite-based bubble propelled micromotors (CFO micromotors) for oxidation of tetracycline (TC) antibiotic, as pharmaceutical model pollutant, via Fentonlike reaction. Tetracycline is one of most widely used antibiotic for human and veterinary healthcare[41]. The CFO micromotors function as self-propelled heterogeneous Fenton-like catalysts in presence of hydrogen peroxide, which acts as both fuel for micromotors propulsion and reagent for Fenton-like oxidation of the antibiotic pollutant. Furthermore, CFO micromotors are also ferromagnetic in nature, which makes it possible to easily recover the micromotors after the completion of degradation process.

\section{Experimental}

\subsection{Synthesis and characterization of micromotors}

CFO micromotors were fabricated from cobalt ferrite nanoparticles. Cobalt ferrite nanoparticles were synthesized by solvothermal method using cobalt acetate (CoAc, sigma 403024) and Iron(III) chloride hexahydrate $\left(\mathrm{FeCl}_{3} \cdot 6 \mathrm{H}_{2} \mathrm{O}\right.$, sigma $\mathrm{F} 2877$ ) as the precursors. First, $0.92 \mathrm{~g} \mathrm{CoAc}$ and $1 \mathrm{~g} \mathrm{FeCl}_{3} \cdot 6 \mathrm{H}_{2} \mathrm{O}$ were dissolved in $30 \mathrm{ml}$ of ethylene glycol (sigma 324558 ). Then, $2.78 \mathrm{mg}$ of sodium acetate (NaAc, sigma S8750) and $1 \mathrm{ml}$ of polyethylene glycol (PEG 200, Alfa Aesar B21918) as the stabilizer were added to the previous mix. The prepared solution was transferred into $50 \mathrm{ml}$ Teflon lined hydrothermal autoclave and heated to $170^{\circ} \mathrm{C}$ for 15 hours. After the reaction was completed the reactor was cool down to room temperature and then the content was transferred into a beaker. The nanoparticle suspension obtained was washed multiple times with isopropanol (Panreac 211090) to remove ethylene glycol and unreacted precursor molecules. Then, the resulting suspension was dried using an oven at $80^{\circ} \mathrm{C}$ for 15 hours. The drying process induces the aggregation of the cobalt ferrite nanoparticles into few hundred-micrometers sizes of 
microparticles. These microparticles were manually grounded and sieved to obtain CFO micromotors with the size under 100 micrometers.

A diluted suspension of the cobalt ferrite nanoparticles was dried overnight at room temperature on a copper grid to prepare samples for the transmission electron microscopy (TEM) analysis. JEOL Centurio model was used to obtain images of nanoparticles. Electron energy loss spectroscopy (EELS) (Gatan GIF ERS electron energy-loss spectrometer)) coupled with TEM was used to quantify ratio of Co and Fe present in the nanoparticles. The scanning electron microscope (SEM) images of CFO micromotors were obtained using a FEI NOVA NanoSEM 230 system. The crystalline structure of micromotors were further characterized by using the Bruker D8 Advance diffractometer equipped with a Cu Ka radiation (1.5417 $\AA$ ) source, a LYNXEYE super speed detector and a Ni filter.

Swimming behavior of the CFO micromotors was analyzed using an inverted optical microscope Leica DMI 3000 B equipped with a camera Leica DFC $3000 \mathrm{G}$. A custom-made 3D printed sample holder was prepared to position the glassware under the microscope for observing the swimming of the micromotors in-situ.

\subsection{Antibiotic degradation experiments using CFO micromotors.}

Tetracycline (TC, sigma T7660) was selected as target antibiotic. TC degradation experiments were carried out in a glass beaker containing CFO micromotors in $50 \mathrm{mg} / \mathrm{L} \mathrm{TC}$ solution and $5 \%$ hydrogen peroxide (sigma 31642). In all the experiments, the concentration of the TC was kept constant. To optimize the degradation approach, we studied different parameters. First, the effect of the amount of CFO micromotors in the efficiency of TC degradation was studied. To this end, three different suspensions of CFO micromotors with the concentrations of $0.05 \mathrm{~g} / \mathrm{L}, 0.1 \mathrm{~g} / \mathrm{L}$, and $0.2 \mathrm{~g} / \mathrm{L}$ were used in triplicate. To achieve the optimized $\mathrm{H}_{2} \mathrm{O}_{2}$ concentration for TC degradation using CFO micromotors, four concentrations of $\mathrm{H}_{2} \mathrm{O}_{2}$ were evaluated ( $1 \% \mathrm{w} / \mathrm{w}, 2.5 \% \mathrm{w} / \mathrm{w}, 5 \% \mathrm{w} / \mathrm{w}$ and $7 \% \mathrm{w} / \mathrm{w})$.

The effect of surfactant was studied by using the optimized concentration of $0.1 \mathrm{~g} / \mathrm{L}$ CFO micromotors, $5 \% \mathrm{w} / \mathrm{w} \mathrm{H}_{2} \mathrm{O}_{2}$ and $50 \mathrm{mg} / \mathrm{L} \mathrm{TC}$. Four sets of experiments were carried out $(\mathrm{n}=3)$ containing three different surfactants, sodium dodecyl sulfate triton- $X$ and tween 20 in $1 \% \mathrm{w} / \mathrm{w}$ concentration in three different sets and one without the presence of any surfactant.

In all the degradation experiments, aliquots of the different samples were taken at timed intervals. The concentration of TC in each sample was measured by reading the absorbance value of the sample at $358 \mathrm{~nm}$ using a UV-Vis spectrometer.

\subsection{Identification of hydroxyl radicals.}

Terephthalic acid was used as a probe to investigate the hydroxyl radical generation during the Fentonlike reaction of CFO micromotors. A solution containing terephthalic acid (0.5mM) (sigma 185361), hydrogen peroxide $(5 \% \mathrm{w} / \mathrm{w})$ and CFO micromotors $(0.1 \mathrm{~g} / \mathrm{L})$ was used for the experiment and an identical solution without micromotors was used as control. The presence of 2-hydroxyterephthalic acid was produced by the reaction between terephthalic acid and the generated hydroxyl radical species. This 
product was detected by measuring its fluorescence spectra from $350 \mathrm{~nm}$ to $600 \mathrm{~nm}$ at $320 \mathrm{~nm}$ excitation wavelength using a multimode microplate reader (Tecan Infinite M200 PRO).

\section{Results and discussion}

CFO micromotors were fabricated from the cobalt ferrite nanoparticles, which were synthesized by using cobalt(II) acetate and iron(III) chloride (molar ratio $\mathrm{Co}^{2+} / \mathrm{Fe}^{3+}=0.5$ ) as precursors in ethylene glycol via solvothermal route[42]. The solvent ethylene glycol acts as a reducing agent while sodium acetate and polyethylene glycol are added in the solution to prevent the aggregation of nanoparticles during the synthesis in the liquid phase.

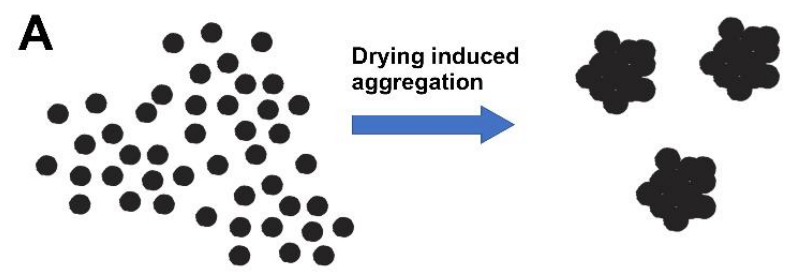

B

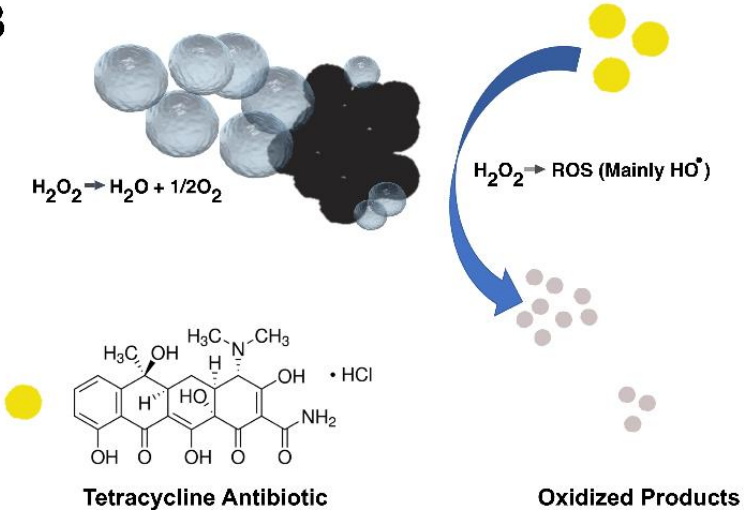

Figure 1. Scheme of the synthesis and mechanism of the cobalt-iron micromotors (CFO micromotors). (A) Synthesis of the CFO micromotors by drying of cobalt ferrite nanoparticles. (B) Degradation of tetracycline (TC) antibiotic by using the self-propelled CFO micromotors.

The synthesized cobalt ferrite nanoparticles were dried to obtain agglomerated micrometer-sized CFO micromotors as depicted in Figure 1A. The CFO micromotors decompose hydrogen peroxide $\left(\mathrm{H}_{2} \mathrm{O}_{2}\right)$ into strongly oxidizing hydroxyl radicals via Fenton-like reaction that can oxidize TC antibiotic into intermediate oxidized products and eventually into carbon dioxide $\left(\mathrm{CO}_{2}\right)$. In addition, the oxygen gas $\left(\mathrm{O}_{2}\right)$ bubbles generated during hydrogen peroxide decomposition induce self-propulsion of the micromotors, which enhances mass transfer and micro-mixing of the solution to further promote the degradation of tetracycline (TC) by the hydroxyl radicals (Figure 1B).

CFO micromotors were characterized by TEM and SEM (Figure $2 \mathrm{~A}, \mathrm{~B} \& \mathrm{C}$ ). Figure 2A displays a TEM image of synthesized cobalt ferrite nanoparticles. It is observed that the diameter of individual nanoparticles is smaller than $100 \mathrm{~nm}$. The cobalt ferrite nanoparticles have tendency to agglomerate because of strong magnetic interactions between the particles, therefore it is hard to prevent their agglomeration, even in 
the suspension form.[42,43] Once the nanoparticles are washed to remove unreacted solvents and surface stabilizer and dried, they strongly adhere with each other and require significant stress to deform.[44,45] The aggregated nanoparticles form microparticles of various sizes, ranging from submicron scale aggregates to a few hundred micrometers. The resulting microparticles were further grinded and sieved to obtain micromotors under 100 micrometers. Figures 2B and C show SEM images of a micrometer sized CFO micromotor obtained from aggregated nanoparticles. The surface of micromotors is rough and porous because of the hierarchical aggregation of the already agglomerated nanoparticles. The roughness and porosity of surface (Figure $2 \mathrm{C}$ ) increase the surface area and provide favorable points for bubble nucleation, necessary for propulsion.

The composition and crystalline structure of the CFO micromotors was analyzed by electron energy loss spectroscopy (EELS) method and XRD respectively (Figure $2 \mathrm{D} \& \mathrm{E}$ ). Individual elements present in the micromotors were quantified to estimate their amount in the structure. Mainly three elements cobalt, iron, and oxygen were detected. The ratio of cobalt and iron elements was found to be 0.5 which is consistent with the ratio of the cobalt and iron precursor used in the synthesis of the micromotors (Figure 2D), confirming the $\mathrm{CoFe}_{2} \mathrm{O}_{4}$ structure. The XRD patterns of the dried $\mathrm{CoFe}_{2} \mathrm{O}_{4}$ micromotors (Figure 2E) evidence a poorly crystalline structure. The (311) and (440) lattice planes correspond to $\mathrm{CoFe}_{2} \mathrm{O}_{4}($ JCPDS 22-1086).

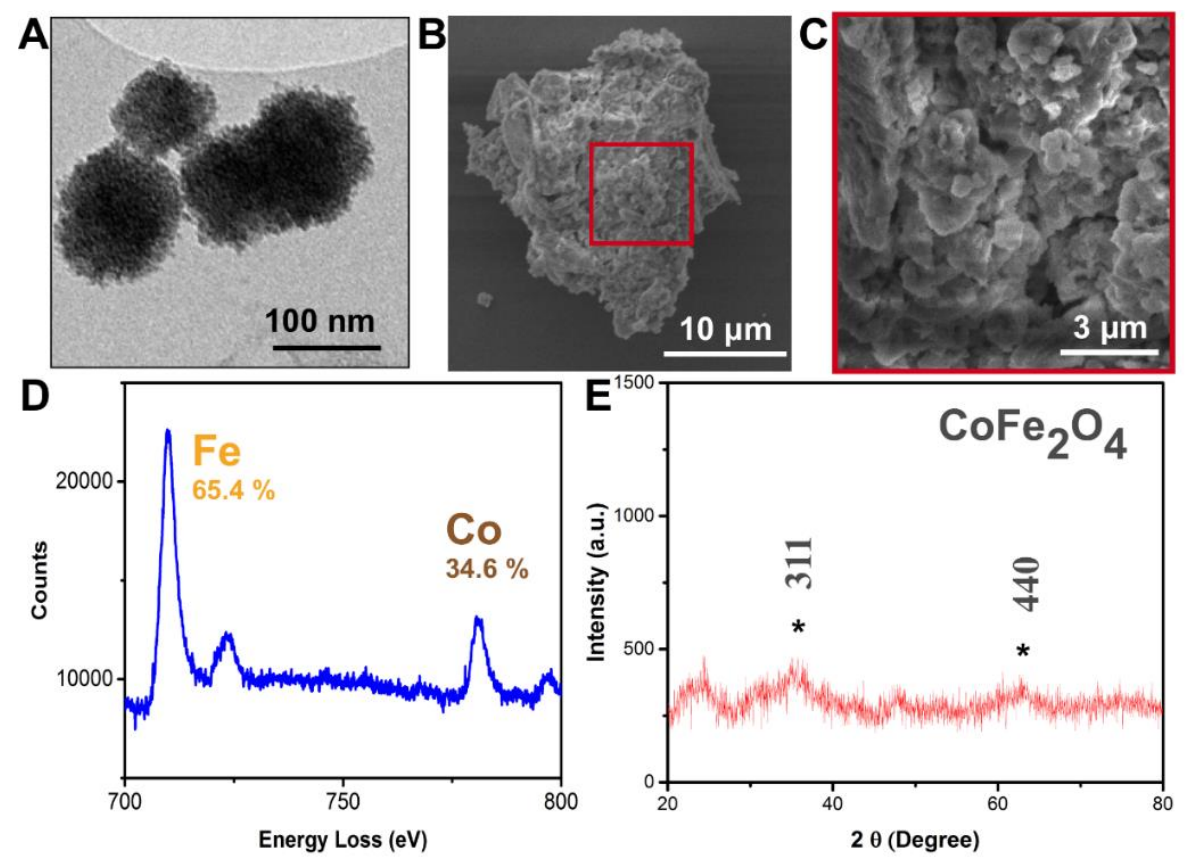

Figure 2. Characterization of the CFO micromotors. (A) TEM image of cobalt ferrite nanoparticles (B) SEM image of a CFO micromotor. (C) SEM image of the surface morphology of a CFO micromotor. (D) Element composition of the CFO micromotors obtained by electron energy loss spectroscopy (EELS). (E) X-ray diffraction (XRD) pattern of CFO micromotors.

The motion of cobalt ferrite nanoparticles and micromotors was investigated by optical microscopy. We observed that the nanoparticles do not exhibit bubble propulsion in $\mathrm{H}_{2} \mathrm{O}_{2}$ solution. Bubbles were only 
observed in the bulk liquid or on the surface of the container due to gas saturation in the sample. On the contrary, in the case of CFO micromotors, they self-propel by the bubbles generated from the decomposition of $\mathrm{H}_{2} \mathrm{O}_{2}$ fuel at their surface because their bigger size and rough surface can provide sufficient nucleation points for bubbles to grow. It has previously been reported that the size limit of the catalytic micromotors for bubble propulsion depends on the catalytic activity, concentration of $\mathrm{H}_{2} \mathrm{O}_{2}$ and various geometrical factors[46]. For instance, platinum coated spherical micromotors can only exhibit bubble propulsion if their diameters are above $10 \mu \mathrm{m}$ [47]. However, tubular micromotors can swim by bubble propulsion even with sub-micrometer diameter due to the confinement of the gas generated in the tubular structure[48]. In the case of CFO micromotors, a precise quantification of their cut-off size for bubble propulsion is difficult to assess due to the asymmetric shape of the micromotors which leads to a different number of nucleation points for the bubble growth and detachment. Figure 3A shows tracking of an individual CFO micromotor swimming in $5 \% \mathrm{w} / \mathrm{w} \mathrm{H}_{2} \mathrm{O}_{2}$ for 10 seconds (Video S1). We observed that the motion of the micromotors was pulsatile and the recoil force of the bubble detachment mainly contributed to their motion. Swarms of CFO micromotors rapidly move around the liquid producing a chaotic swimming behavior (Video S2) that can induce an efficient mixing in the system. Figure 3B illustrates the tracking of four different CFO micromotors swimming for 10 seconds. CFO micromotors can maintain individual swimming direction even though bursting and fusion of the bubbles create often strong drift in the liquid.
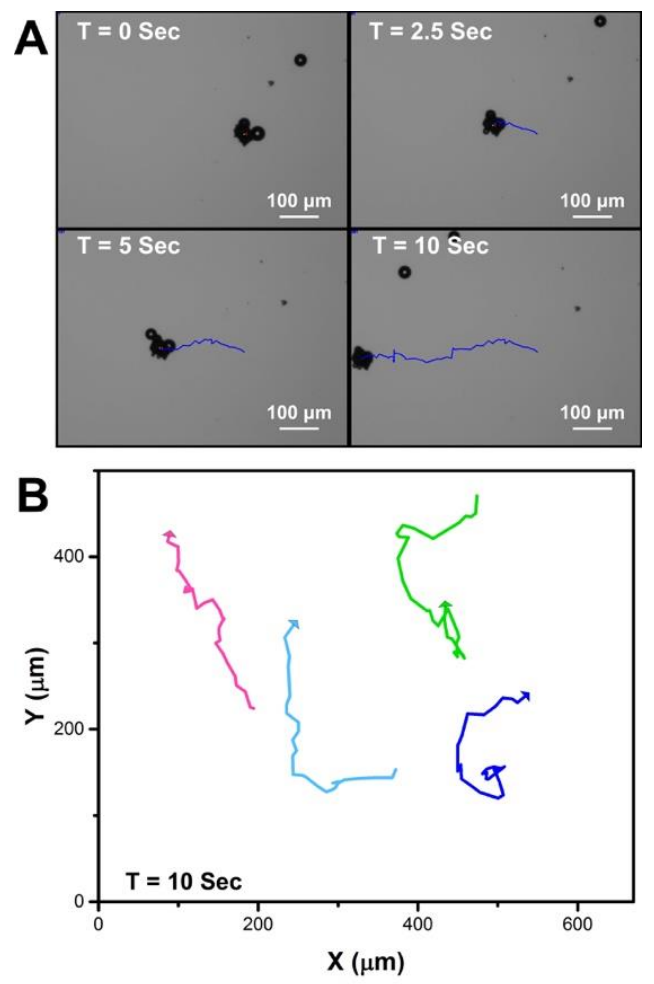

Figure 3. Swimming behavior of CFO micromotors. (A) Tracking of an individual CFO micromotor swimming in $5 \% \mathrm{w} / \mathrm{w} \mathrm{H}_{2} \mathrm{O}_{2}$ concentration. (B) Tracking of multiple CFO micromotors swimming in $5 \% \mathrm{w} / \mathrm{w} \mathrm{H}_{2} \mathrm{O}_{2}$. 
The CFO micromotors were studied for their efficiency towards TC degradation via Fenton-like reaction. First, the micromotors concentration was optimized by using different amounts of CFO micromotors from 0.05 to $0.2 \mathrm{~g} / \mathrm{L}$ in the presence of $5 \% \mathrm{w} / \mathrm{w} \mathrm{H}_{2} \mathrm{O}_{2}$ (Figure $4 \mathrm{~A}$ ). We observed that the degradation rate of TC did not increase above $0.1 \mathrm{~g} / \mathrm{L}$ micromotors. To study the effect of $\mathrm{H}_{2} \mathrm{O}_{2}$, further experiments were carried out using $0.1 \mathrm{~g} / \mathrm{L}$ CFO micromotors and varying the $\mathrm{H}_{2} \mathrm{O}_{2}$ concentration (1\%, 2.5\%, $5 \%$ and $7.5 \% \mathrm{w} / \mathrm{w}$ ). As it is observed in Figure 4B, the TC degradation rate increased by increasing $\mathrm{H}_{2} \mathrm{O}_{2}$ concentration, reaching a maximum at $5 \% \mathrm{w} / \mathrm{w}$ (Figure $4 \mathrm{~B}$ inset). $0.1 \mathrm{~g} / \mathrm{L}$ micromotors and $5 \% \mathrm{w} / \mathrm{w} \mathrm{H}_{2} \mathrm{O}_{2}$ were selected as optimized concentrations and for further experiments to study the effect of surfactants and the generation of hydroxyl radicals.
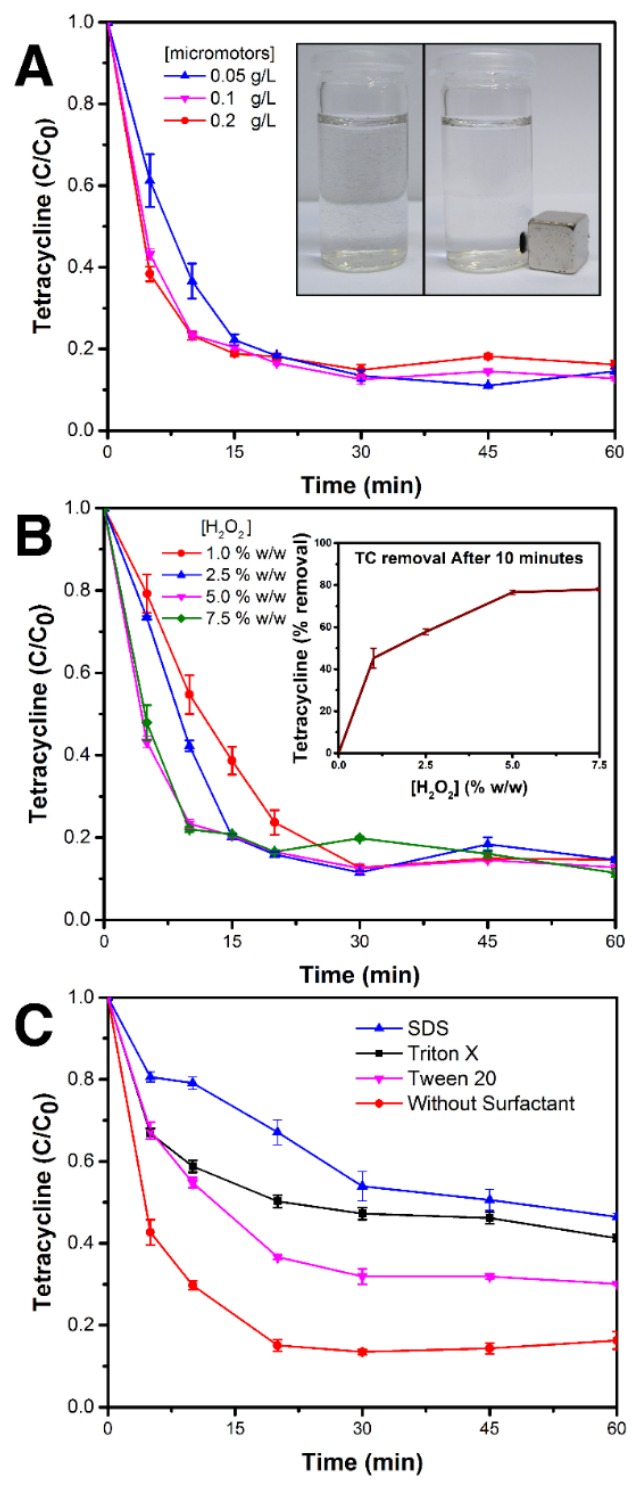

Figure 4. Degradation of TC antibiotic by CFO micromotors. (A) Effect of different amount of CFO micromotors. (Inset: Extraction of micromotors using $\mathrm{NdFeB}$ magnet) (B) Effect of different $\mathrm{H}_{2} \mathrm{O}_{2}$ concentrations. (Inset: $\mathrm{TC}$ removal after 10 minutes of reaction with micromotors at different $\mathrm{H}_{2} \mathrm{O}_{2}$ concentrations). (C) Effect of presence of various surfactants 
Generally, surfactants are added to the solution to improve the overall swimming behavior of the micromotors regarding directionality and speed. Surfactants stabilize the interface of bubbles therefore facilitating their detachment from the micromotors surface, which smoothens the swimming and decreases the drift in the liquid due to fusion and bursting of bubbles[49]. In certain cases, surfactants are even necessary to achieve motion of micromotors.[50] Recently, it has been reported that addition of surfactants improves pollutant removal efficiency of micromotors because of the improvement in the swimming behavior and adsorptive separation of pollutants on the bubbles.[15,34,51] However, surfactants increase the organic matter of the wastewater and can be toxic, requiring further treatment such as electrocoagulation processes[52,53]. We studied the performance of CFO micromotors for TC antibiotic degradation in the presence of various surfactants, such as sodium dodecyl sulfate (SDS), triton$X$ and Tween 20 and without the addition of surfactant. CFO micromotors performed better ( 84\%) for removal of TC when surfactants were not added in the system. However, the presence of surfactants severely affected the performance of degradation of TC by the CFO micromotors (Figure 4C). Indeed, it was observed that SDS ( $54 \%$ removal) decreased the efficiency of TC degradation more significantly than Tween 20 and Triton- $X$ ( $70 \%$ and $\sim 59 \%$ removal respectively). The extra organic load added by the presence of the surfactant can competitively consume the reactive oxygen species produced by the Fenton-like reaction. Furthermore, surfactants form micellar structure around TC molecules that may oxidize first and prevent access of hydroxyl radicals to TC. This effect can be enhanced for anionic surfactant like SDS due to its electrostatic interaction with TC[54].

The degradation efficiency of TC by the CFO micromotors was compared with control experiments using only $\mathrm{H}_{2} \mathrm{O}_{2}$ without the addition of the CFO micromotors and the TC degradation by the micromotors without the addition of $\mathrm{H}_{2} \mathrm{O}_{2}$. As shown in Figure $5 \mathrm{~A}$, only a small decrease in the characteristic absorbance peak of TC in both control experiments were observed, which can be attributed to the oxidative nature of the $\mathrm{H}_{2} \mathrm{O}_{2}$ and adsorption of TC on the CFO micromotors. TC degradation was minimal in the control experiments while in the presence of swimming CFO micromotors most of the antibiotic was degraded from the solution after 10 min of the Fenton-like reaction, being the characteristic absorbance peak of TC negligible. 

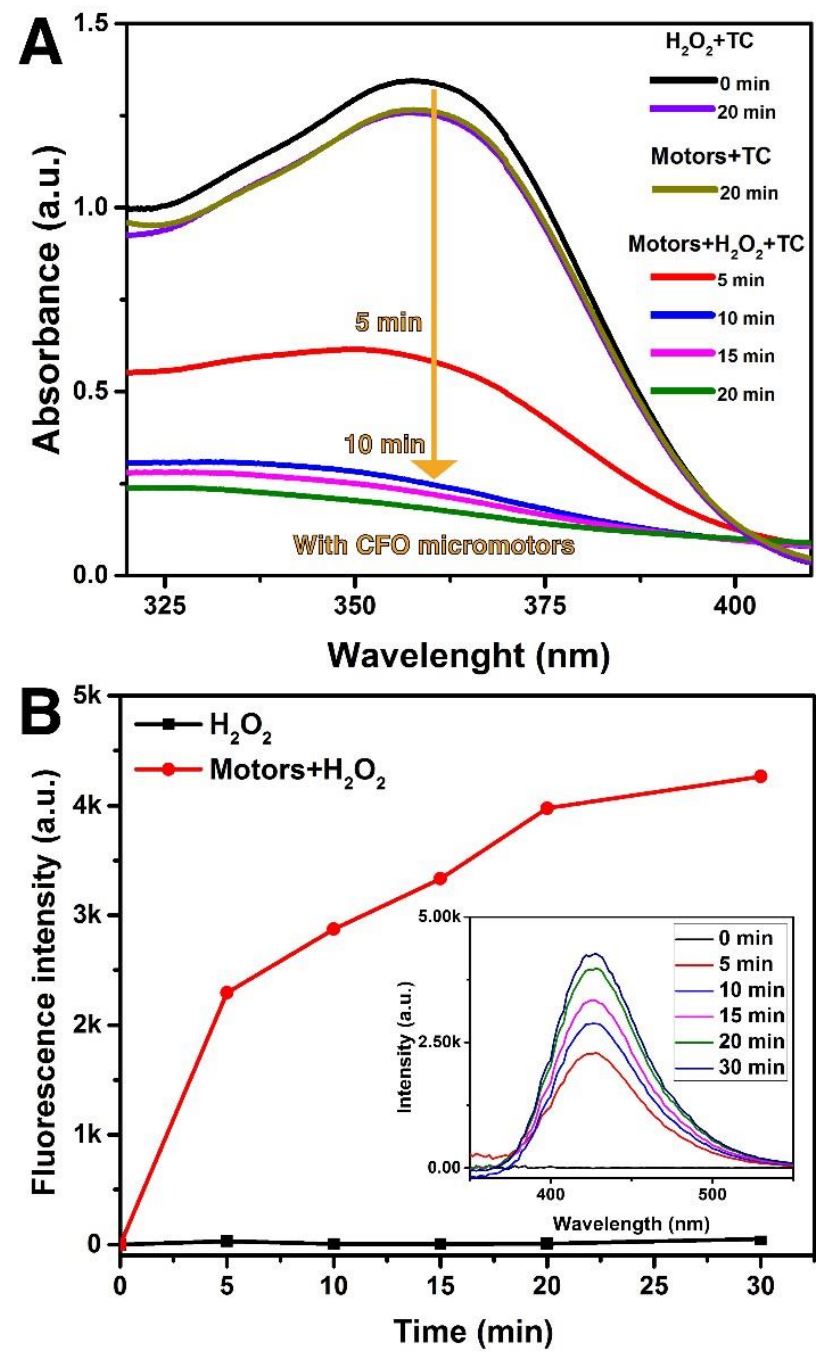

Figure 5. (A) Degradation of TC by the CFO micromotors approach at optimized $\mathrm{H}_{2} \mathrm{O}_{2}$ concentration (5\%) and CFO micromotors amount $(0.1 \mathrm{~g} / \mathrm{L}$ ) over time. (B) Hydroxyl radical generated during the swimming of CFO micromotors in $\mathrm{H}_{2} \mathrm{O}_{2}$ probed by fluorescence intensity measurement of 2-hydroxyterephthalic acid converted from terephthalic acid. (Inset: fluorescence spectra scan over time).

The degradation pathway of TC was previously studied by other researchers giving a general idea of its possible mineralization pathways [55]. The mechanism includes oxidation of the methyl and amino groups attached to the aromatic rings, followed by the ring opening reactions that subsequently become oxidized into the short chain carboxylic acids before mineralization into carbon dioxide. Because of the complex structure of the TC molecules, many intermediate molecules can be produced during the oxidative removal. Hydroxyl radicals are considered the key oxidative radicals generated in the Fenton-like reaction that are mainly responsible for such mineralization process. To verify the generation of the hydroxyl radical by CFO micromotors, we used terephthalic acid as probe molecule, that acts as selective scavenger of hydroxyl radicals and converts into 2-hydroxyterephthalic, which is fluorescent. The increase in 2hydroxyterephthalic acid concentration was measured by fluorescence spectrometry. Figure 5B displays 
the increase in the fluorescence signal of 2-hydroxyterephthalic acid over time when the CFO micromotors are swimming in mixture of $\mathrm{H}_{2} \mathrm{O}_{2}$ and terephthalic acid solution, thus confirming the presence of the hydroxyl radicals. Fluorescence signal of 2-hydroxyterephthalic acid rapidly increases over the time during the Fenton-like reaction of CFO micromotors indicating an increase in the generation of hydroxyl radicals. Hydroxy radical production rate slows down after 20 minutes of reaction which can be attributed to depletion in both, $\mathrm{H}_{2} \mathrm{O}_{2}$ and terephthalic acid concentrations.

Fenton reaction mechanism, proposed by Haber and Weiss, can explain both the production of hydroxyl radicals for organic degradation (Eq.1) and oxygen bubble for self-propulsion in the ferrous ions recycling step (Eq. 3).

$$
\begin{array}{lc}
\mathrm{Fe}_{\text {surface }}^{2+}+\mathrm{H}_{2} \mathrm{O}_{2} \rightarrow \mathrm{Fe}_{\text {surface }}^{3+}+{ }^{+} \mathrm{OH}+{ }^{-} \mathrm{OH} & \text { Eq. } 1 \\
\mathrm{H}_{2} \mathrm{O}_{2}+\cdot \mathrm{OH} \rightarrow \mathrm{H}_{2} \mathrm{O}+{ }^{\cdot} \mathrm{OOH} & E q .2 \\
\mathrm{Fe}_{\text {surface }}^{3+}+\cdot \mathrm{OOH} \rightarrow \mathrm{Fe}_{\text {surface }}^{2+}+\mathrm{H}^{+}+\mathrm{O}_{2} & \text { Eq. } 3
\end{array}
$$

Presence of cobalt in the catalyst structure can also activate hydrogen peroxide for increased production of hydroxyl radicals, thus increasing the efficiency of the catalyst (Eq. 4).

$$
\begin{array}{ll}
\mathrm{Co}_{\text {surface }}^{2+}+\mathrm{H}_{2} \mathrm{O}_{2} \rightarrow \mathrm{Co}_{\text {surface }}^{3+}+{ }^{+} \mathrm{OH}+{ }^{-} \mathrm{OH} & \text { Eq. } 4 \\
\mathrm{Co}_{\text {surface }}^{3+}+\mathrm{Fe}_{\text {surface }}^{2+} \rightarrow \mathrm{Co}_{\text {surface }}^{2+}+\mathrm{Fe}_{\text {surface }}^{3+} & \text { Eq. } 5
\end{array}
$$

$\mathrm{Co}^{3+}$ can be recycled back to $\mathrm{Co}^{2+}$ by $\mathrm{Fe}^{2+}$ because of the thermodynamically favorable redox reaction to accelerate decomposition of hydrogen peroxide[56] (Eq. 5). Apart from above mentioned radical mediated production of oxygen gas, surface oxygen vacancies present in the metal oxides can also decompose hydrogen peroxide directly into oxygen and water without producing intermediate hydroxyl radicals [57]. However, the detection of hydroxyl radicals suggests the involvement of radical mediated pathways for CFO micromotors for both the degradation of TC and generation of oxygen gas for selfpropulsion.

\section{Conclusions}

We have fabricated inexpensive CFO based micromotors by nanoparticle drying process, using easily scalable synthesis methods. The CFO micromotors can swim without the addition of surfactants in the system and efficiently degrade antibiotics from wastewater. Furthermore, we observed that the presence of the surfactants decreases the efficiency of CFO micromotors. We have also verified the production of hydroxyl radicals to support the oxidation mechanism of TC by CFO micromotors. Since the hydroxyl radicals are non-selective oxidants, these micromotors can potentially be used for wide range of pharmaceutical and other organic pollutants. We believe that CFO micromotors do not only demonstrate the usability of self-propelled micromotors for wastewater cleaning, but also show an improvement of 
previously reported systems, since they are composed of cheap materials and do not require the addition of surfactants, which are an extra organic load on the water.

\section{Conflicts of interest}

There are no conflicts of interest to declare.

\section{Acknowledgements}

The research leading to these results has received funding from the ERC under the European Union's Seventh Framework Program (FP7/20072013)/ERC grant agreement no. 311529 (LT-NRBS) and ERC-2015PoC/Project ID: 713608 (MICROCLEANERS)). Authors would like to thank to S. Murcia-López and T. Andreu from the IREC Barcelona for the XRD measurements of the materials and Mr. Kersten Hahn for assisting with the TEM images and EELS analysis. D. V. acknowledges financial support provided by the ERC under Horizon 2020's Marie Skłodowska-Curie Actions COFUND scheme [Grant no. 712754] and by the Severo Ochoa programme of MICINN [Grant SEV-2014-0425]. S.S. thanks the Spanish MINECO for grants CTQ2015-68879-R (MICRODIA) and CTQ2015-72471-EXP (Enzwim).

\section{References}

[1] J. Wang, Nanomachines: fundamentals and applications, Wiley-VCH, Weinheim, 2013.

[2] S. Sánchez, L. Soler, J. Katuri, Chemically Powered Micro- and Nanomotors, Angew. Chem. Int. Ed. 54 (2015) 1414-1444. doi:10.1002/anie.201406096.

[3] H. Wang, M. Pumera, Fabrication of Micro/Nanoscale Motors, Chem. Rev. 115 (2015) 8704-8735.

[4] J. Katuri, X. Ma, M.M. Stanton, S. Sánchez, Designing Micro- and Nanoswimmers for Specific Applications, Acc. Chem. Res. 50 (2017) 2-11. doi:10.1021/acs.accounts.6b00386.

[5] X.-Z. Chen, M. Hoop, F. Mushtaq, E. Siringil, C. Hu, B.J. Nelson, S. Pané, Recent developments in magnetically driven micro- and nanorobots, Appl. Mater. Today. 9 (2017) 37-48. doi:10.1016/j.apmt.2017.04.006.

[6] B. Jurado-Sánchez, A. Escarpa, Janus Micromotors for Electrochemical Sensing and Biosensing Applications: A Review, Electroanalysis. 29 (2017) 14-23. doi:10.1002/elan.201600567.

[7] S. Campuzano, D. Kagan, J. Orozco, J. Wang, Motion-driven sensing and biosensing using electrochemically propelled nanomotors, Analyst. 136 (2011) 4621-4630. doi:10.1039/C1AN15599G.

[8] B. Jurado-Sánchez, A. Escarpa, Milli, micro and nanomotors: Novel analytical tools for real-world applications, TrAC Trends Anal. Chem. 84 (2016) 48-59. doi:10.1016/j.trac.2016.03.009.

[9] W. Xi, A.A. Solovev, A.N. Ananth, D.H. Gracias, S. Sanchez, O.G. Schmidt, Rolled-up magnetic microdrillers: towards remotely controlled minimally invasive surgery, Nanoscale. 5 (2013) 1294-1297. doi:10.1039/C2NR32798H. 
[10] S.K. Srivastava, M. Medina-Sánchez, B. Koch, O.G. Schmidt, Medibots: Dual-Action Biogenic Microdaggers for Single-Cell Surgery and Drug Release, Adv. Mater. 28 (2016) 832-837. doi:10.1002/adma.201504327.

[11] J. Pokki, J. Parmar, O. Ergeneman, H. Torun, M. Guerrero, E. Pellicer, J. Sort, S. Pané, B.J. Nelson, Mobility-Enhancing Coatings for Vitreoretinal Surgical Devices: Hydrophilic and Enzymatic Coatings Investigated by Microrheology, ACS Appl. Mater. Interfaces. 7 (2015) 22018-22028. doi:10.1021/acsami.5b06937.

[12] W. Gao, J. Wang, Synthetic micro/nanomotors in drug delivery, Nanoscale. 6 (2014) 10486-10494. doi:10.1039/C4NR03124E.

[13] F. Peng, Y. Tu, D.A. Wilson, Micro/nanomotors towards in vivo application: cell, tissue and biofluid, Chem. Soc. Rev. (2017). doi:10.1039/C6CS00885B.

[14] M. Guix, J. Orozco, M. García, W. Gao, S. Sattayasamitsathit, A. Merkoçi, A. Escarpa, J. Wang, Superhydrophobic Alkanethiol-Coated Microsubmarines for Effective Removal of Oil, ACS Nano. 6 (2012) 4445-4451. doi:10.1021/nn301175b.

[15] L. Soler, V. Magdanz, V.M. Fomin, S. Sanchez, O.G. Schmidt, Self-Propelled Micromotors for Cleaning Polluted Water, ACS Nano. 7 (2013) 9611-9620. doi:10.1021/nn405075d.

[16] L. Soler, S. Sánchez, Catalytic nanomotors for environmental monitoring and water remediation, Nanoscale. 6 (2014) 7175-7182.

[17] W. Gao, J. Wang, The environmental impact of micro/nanomachines: a review, Acs Nano. 8 (2014) 3170-3180.

[18] M. Safdar, J. Simmchen, J. Jänis, Light-driven micro- and nanomotors for environmental remediation, Environ. Sci. Nano. 4 (2017) 1602-1616. doi:10.1039/C7EN00367F.

[19] J. Orozco, B. Jurado-Sánchez, G. Wagner, W. Gao, R. Vazquez-Duhalt, S. Sattayasamitsathit, M. Galarnyk, A. Cortés, D. Saintillan, J. Wang, Bubble-Propelled Micromotors for Enhanced Transport of Passive Tracers, Langmuir. 30 (2014) 5082-5087. doi:10.1021/la500819r.

[20] D. Vilela, J. Parmar, Y. Zeng, Y. Zhao, S. Sánchez, Graphene-Based Microbots for Toxic Heavy Metal Removal and Recovery from Water, Nano Lett. 16 (2016) 2860-2866. doi:10.1021/acs.nanolett.6b00768.

[21] D.A. Uygun, B. Jurado-Sánchez, M. Uygun, J. Wang, Self-propelled chelation platforms for efficient removal of toxic metals, Environ. Sci. Nano. 3 (2016) 559-566. doi:10.1039/C6EN00043F.

[22] J. Orozco, G. Cheng, D. Vilela, S. Sattayasamitsathit, R. Vazquez-Duhalt, G. Valdés-Ramírez, O.S. Pak, A. Escarpa, C. Kan, J. Wang, Micromotor-Based High-Yielding Fast Oxidative Detoxification of Chemical Threats, Angew. Chem. Int. Ed. 52 (2013) 13276-13279. 
[23] J. Parmar, D. Vilela, E. Pellicer, D. Esqué-de los Ojos, J. Sort, S. Sánchez, Reusable and Long-Lasting Active Microcleaners for Heterogeneous Water Remediation, Adv. Funct. Mater. 26 (2016) 4152-4161. doi:10.1002/adfm.201600381.

[24] J. Orozco, L.A. Mercante, R. Pol, A. Merkoci, Graphene-based Janus micromotors for the dynamic removal of pollutants, J. Mater. Chem. A. (2016). doi:10.1039/C5TA09850E.

[25] M. Xuan, X. Lin, J. Shao, L. Dai, Q. He, Motion-Based, High-Yielding, and Fast Separation of Different Charged Organics in Water, ChemPhysChem. 16 (2015) 147-151.

[26] F. Mushtaq, A. Asani, M. Hoop, X.-Z. Chen, D. Ahmed, B.J. Nelson, S. Pané, Highly Efficient Coaxial TiO2-PtPd Tubular Nanomachines for Photocatalytic Water Purification with Multiple Locomotion Strategies, Adv. Funct. Mater. 26 (2016) 6995-7002. doi:10.1002/adfm.201602315.

[27] F. Mou, D. Pan, C. Chen, Y. Gao, L. Xu, J. Guan, Magnetically Modulated Pot-Like MnFe2O4 Micromotors: Nanoparticle Assembly Fabrication and their Capability for Direct Oil Removal, Adv. Funct. Mater. 25 (2015) 6173-6181. doi:10.1002/adfm.201502835.

[28] F. Mushtaq, M. Guerrero, M.S. Sakar, M. Hoop, A.M. Lindo, J. Sort, X. Chen, B.J. Nelson, E. Pellicer, S. Pane, Magnetically driven $\mathrm{Bi} 2 \mathrm{O} 3 / \mathrm{BiOCl}$-based hybrid microrobots for photocatalytic water remediation, J. Mater. Chem. A. 3 (2015) 23670-23676. doi:10.1039/C5TA05825B.

[29] D. Vilela, M.M. Stanton, J. Parmar, S. Sánchez, Microbots Decorated with Silver Nanoparticles Kill Bacteria in Aqueous Media, ACS Appl. Mater. Interfaces. (2017). doi:10.1021/acsami.7b03006.

[30] M. Hoop, Y. Shen, X.-Z. Chen, F. Mushtaq, L.M. Iuliano, M.S. Sakar, A. Petruska, M.J. Loessner, B.J. Nelson, S. Pané, Magnetically Driven Silver-Coated Nanocoils for Efficient Bacterial Contact Killing, Adv. Funct. Mater. 26 (2016) 1063-1069. doi:10.1002/adfm.201504463.

[31] J.A.M. Delezuk, D.E. Ramírez-Herrera, B.E.-F. de Ávila, J. Wang, Chitosan-based water-propelled micromotors with strong antibacterial activity, Nanoscale. 9 (2017) 2195-2200. doi:10.1039/C6NR09799E.

[32] Y. Ge, M. Liu, L. Liu, Y. Sun, H. Zhang, B. Dong, Dual-Fuel-Driven Bactericidal Micromotor, NanoMicro Lett. 8 (2016) 157-164. doi:10.1007/s40820-015-0071-3.

[33] H. Wang, G. Zhao, M. Pumera, Beyond Platinum: Bubble-Propelled Micromotors Based on Ag and MnO2 Catalysts, J. Am. Chem. Soc. 136 (2014) 2719-2722. doi:10.1021/ja411705d.

[34] W.Z. Teo, R. Zboril, I. Medrik, M. Pumera, Fe0 Nanomotors in Ton Quantities (1020 Units) for Environmental Remediation, Chem. - Eur. J. 22 (2016) 4789-4793. doi:10.1002/chem.201504912.

[35] O.M. Wani, M. Safdar, N. Kinnunen, J. Jänis, Dual Effect of Manganese Oxide Micromotors: Catalytic Degradation and Adsorptive Bubble Separation of Organic Pollutants, Chem. - Eur. J. 22 (2016) 1244-1247. doi:10.1002/chem.201504474. 
[36] W. Barb, J. Baxendale, P. George, K. Hargrave, Reactions of ferrous and ferric ions with hydrogen peroxide, Nature. 163 (1949) 692-694.

[37] M. Klavarioti, D. Mantzavinos, D. Kassinos, Removal of residual pharmaceuticals from aqueous systems by advanced oxidation processes, Environ. Int. 35 (2009) 402-417.

[38] I. Sirés, E. Brillas, Remediation of water pollution caused by pharmaceutical residues based on electrochemical separation and degradation technologies: A review, Environ. Int. 40 (2012) 212-229. doi:10.1016/j.envint.2011.07.012.

[39] Y. Luo, W. Guo, H.H. Ngo, L.D. Nghiem, F.I. Hai, J. Zhang, S. Liang, X.C. Wang, A review on the occurrence of micropollutants in the aquatic environment and their fate and removal during wastewater treatment, Sci. Total Environ. 473 (2014) 619-641. doi:10.1016/j.scitotenv.2013.12.065.

[40] N. Le-Minh, S.J. Khan, J.E. Drewes, R.M. Stuetz, Fate of antibiotics during municipal water recycling treatment processes, Water Res. 44 (2010) 4295-4323. doi:10.1016/j.watres.2010.06.020.

[41] R. Daghrir, P. Drogui, Tetracycline antibiotics in the environment: a review, Environ. Chem. Lett. 11 (2013) 209-227. doi:10.1007/s10311-013-0404-8.

[42] H. Deng, X. Li, Q. Peng, X. Wang, J. Chen, Y. Li, Monodisperse Magnetic Single-Crystal Ferrite Microspheres, Angew. Chem. Int. Ed. 44 (2005) 2782-2785. doi:10.1002/anie.200462551.

[43] Q. Dai, M. Lam, S. Swanson, R.-H.R. Yu, D.J. Milliron, T. Topuria, P.-O. Jubert, A. Nelson, Monodisperse Cobalt Ferrite Nanomagnets with Uniform Silica Coatings, Langmuir. 26 (2010) 1754617551. doi:10.1021/la103042q.

[44] K. Kendall, T.P. Weihs, Adhesion of nanoparticles within spray dried agglomerates, J. Phys. Appl. Phys. 25 (1992) A3. doi:10.1088/0022-3727/25/1A/002.

[45] A. Maskara, D.M. Smith, Agglomeration during the Drying of Fine Silica Powders, Part II: The Role of Particle Solubility, J. Am. Ceram. Soc. 80 (1997) 1715-1722. doi:10.1111/j.1151-2916.1997.tb03044.x.

[46] X. Ma, S. Jang, M.N. Popescu, W.E. Uspal, A. Miguel-López, K. Hahn, D.-P. Kim, S. Sánchez, Reversed Janus Micro/Nanomotors with Internal Chemical Engine, ACS Nano. 10 (2016) 8751-8759. doi:10.1021/acsnano.6b04358.

[47] M. Manjare, B. Yang, Y.-P. Zhao, Bubble Driven Quasioscillatory Translational Motion of Catalytic Micromotors, Phys. Rev. Lett. 109 (2012) 128305.

[48] S. Sanchez, A.A. Solovev, S.M. Harazim, C. Deneke, Y. Feng Mei, O.G. Schmidt, The smallest manmade jet engine, Chem. Rec. 11 (2011) 367-370. doi:10.1002/tcr.201100010.

[49] J. Simmchen, V. Magdanz, S. Sanchez, S. Chokmaviroj, D. Ruiz-Molina, A. Baeza, O.G. Schmidt, Effect of surfactants on the performance of tubular and spherical micromotors - a comparative study, RSC Adv. 4 (2014) 20334-20340. doi:10.1039/C4RA02202E. 
[50] H. Wang, G. Zhao, M. Pumera, Crucial Role of Surfactants in Bubble-Propelled Microengines, J. Phys. Chem. C. 118 (2014) 5268-5274. doi:10.1021/jp410003e.

[51] O.M. Wani, M. Safdar, N. Kinnunen, J. Jänis, Dual Effect of Manganese Oxide Micromotors: Catalytic Degradation and Adsorptive Bubble Separation of Organic Pollutants, Chem. Eur. J. (2015).

[52] M. Lechuga, M. Fernández-Serrano, E. Jurado, J. Núñez-Olea, F. Ríos, Acute toxicity of anionic and non-ionic surfactants to aquatic organisms, Ecotoxicol. Environ. Saf. 125 (2016) 1-8. doi:10.1016/j.ecoenv.2015.11.027.

[53] E. Yüksel, I.A. Şengil, M. Özacar, The removal of sodium dodecyl sulfate in synthetic wastewater by peroxi-electrocoagulation method, Chem. Eng. J. 152 (2009) 347-353. doi:10.1016/j.cej.2009.04.058.

[54] X. Dang, C. Hu, Y. Wei, W. Chen, S. Hu, Sensitivity Improvement of the Oxidation of Tetracycline at Acetylene Black Electrode in the Presence of Sodium Dodecyl Sulfate, Electroanalysis. 16 (2004) 19491955. doi:10.1002/elan.200403049.

[55] N. Barhoumi, H. Olvera-Vargas, N. Oturan, D. Huguenot, A. Gadri, S. Ammar, E. Brillas, M.A. Oturan, Kinetics of oxidative degradation/mineralization pathways of the antibiotic tetracycline by the novel heterogeneous electro-Fenton process with solid catalyst chalcopyrite, Appl. Catal. B Environ. 209 (2017) 637-647. doi:10.1016/j.apcatb.2017.03.034.

[56] R.C.C. Costa, M.F.F. Lelis, L.C.A. Oliveira, J.D. Fabris, J.D. Ardisson, R.R.V.A. Rios, C.N. Silva, R.M. Lago, Novel active heterogeneous Fenton system based on Fe3-xMxO4 (Fe, $\mathrm{Co}, \mathrm{Mn}, \mathrm{Ni}$ ): The role of M2+ species on the reactivity towards H2O2 reactions, J. Hazard. Mater. 129 (2006) 171-178. doi:10.1016/j.jhazmat.2005.08.028.

[57] J. He, X. Yang, B. Men, D. Wang, Interfacial mechanisms of heterogeneous Fenton reactions catalyzed by iron-based materials: A review, J. Environ. Sci. 39 (2016) 97-109. doi:10.1016/j.jes.2015.12.003. 